\title{
Targeting of $\alpha_{\mathrm{v}}$ integrins interferes with FAK activation and smooth muscle cell migration and invasion
}

\author{
Jeeva Varadarajulu ${ }^{\mathrm{a}, 1}$, Martin Laser ${ }^{\mathrm{b}, 1}$, Markus Hupp ${ }^{\mathrm{b}}$, Rongxue Wu ${ }^{\mathrm{b}}$, \\ Christof R. Hauck ${ }^{a, *}$ \\ ${ }^{a}$ ZINF, Universität Würzburg, Röntgenring 11, 97070 Würzburg, Germany \\ ${ }^{\mathrm{b}}$ Medizinische Universitätsklinik, Abteilung Innere Medizin, Josef-Schneider-Str. 2, 97080 Würzburg, Germany
}

Received 15 March 2005

Available online 5 April 2005

\begin{abstract}
Aberrant migration of smooth muscle cells (SMCs) is a key feature of restenosis. Since extracellular matrix proteins and their receptors of the integrin family play a critical role in this process, it is instrumental to understand their contribution to cell migration and invasive motility of SMC on the molecular level. Therefore, we investigated the role of $\alpha_{\mathrm{v}}$-containing integrins expressed by primary human coronary artery smooth muscle cells (hCASMCs) in vitronectin (VN)-initiated signaling events and cell migration. In hCASMC plated on $\mathrm{VN}, \alpha_{\mathrm{v}}$-containing integrins were localized at focal adhesion sites. Haptotactic stimulation through VN led to a dose-dependent increase in cell migration and concomitantly to enhanced tyrosine phosphorylation of focal adhesion kinase. Both events were completely blocked by a specific inhibitor of integrin $\alpha_{v}$. Additionally, the integrin $\alpha_{v}$ inhibitor abolished PDGFBB-stimulated chemotactic migration. Confocal microscopy confirmed the increased tyrosine phosphorylation at VN-initiated focal contact sites in hCASMC, that was abolished upon $\alpha_{\mathrm{v}}$ inhibition. In vitro invasion of hCASMC was severely compromised in the presence of the integrin $\alpha_{v}$ inhibitor paralleled by decreased levels of secreted matrix metalloprotease 2 (MMP-2). Together, integrin $\alpha_{\mathrm{v}}$ inhibition abrogates tyrosine phosphorylation at focal adhesion sites and diminishes MMP-2 secretion leading to reduced migration and invasion of hCASMCs.
\end{abstract}

(C) 2005 Elsevier Inc. All rights reserved.

Keywords: Cell adhesion; Extracellular matrix proteins; Tyrosine phosphorylation; Vitronectin

Percutaneous transluminal coronary angioplasty (PTCA) in combination with coronary stent implantation is an accepted treatment of angina pectoris or after myocardial infarction. However, despite recent progress in stent design and despite the introduction of drug-eluting stents, restenosis, the re-narrowing of the dilated blood vessel, is still a major drawback of PTCA. Restenosis occurs in approximately $10-20 \%$ of the patients, depending on their individual cardiovascular risk profile $[1,2]$.

The pathophysiology of restenosis after PTCA or stent implantation is well described (for review [3]). Immedi-

\footnotetext{
* Corresponding author. Fax: +49 931312578

E-mail address: christof.hauck@mail.uni-wuerzburg.de (C.R. Hauck).

${ }^{1}$ These authors contributed equally.
}

ately after the intervention, an early inflammatory response characterized by the recruitment and adhesion of leucocytes, macrophages, and activated platelets to the site of the vessel injury can be observed. In addition to various growth factors and cytokines released locally by resident and recruited cells, plasma proteins such as vitronectin, osteopontin, and fibronectin are deposited at the site of the lesion [4-7]. A combination of these chemotactic and haptotactic stimuli drives the proliferation and migration of vascular cells, mainly smooth muscle cells (SMCs), leading to their accumulation in the newly formed neointima. The migration of SMC from the media to the blood vessel lumen as well as their phenotypic change to a matrix protein secreting cell type contribute to the re-narrowing of the vessel. The result of this 
exaggerated healing response is a restenotic lesion, most often accompanied by a reappearance of the clinical symptoms requiring revascularization.

The current concept of applying cytostatic compounds (e.g., sirolimus or paclitaxel) by means of drug-eluting stents can significantly reduce restenosis formation [8]. However, restenosis cannot be inhibited in all patients, particularly if they belong to a group with higher risk for restenosis. Further, the unselective cytostatic effects of these compounds also suppress endothelial cell migration resulting in delayed or incomplete healing with a higher risk for late thrombosis [9]. Therefore, a more selective approach to control the excess healing response of vascular SMC would be desirable.

An attractive approach is the direct inhibition of SMC migration by targeting the respective extracellular matrix receptors responsible for adhesion and migration of SMC. In particular integrins, heterodimeric membrane glycoproteins that build up more than 30 distinct receptors, specify the substrate binding properties of mammalian cells and several integrins are expressed by SMC. While integrins $\alpha_{5} \beta_{1}$ and $\alpha_{2 / 3} \beta_{1}$ are most likely responsible for SMC adhesion to fibronectin and collagen, the integrins $\alpha_{v} \beta_{3}$ and $\alpha_{v} \beta_{5}$ are cellular receptors for vitronectin and osteopontin [10]. Accordingly, several in vivo and in vitro studies suggested that the application of small peptide inhibitors or antibodies against integrin $\alpha_{\mathrm{v}} \beta_{3}$ (such as Abciximab) prevents SMC migration $[11,12]$ and significantly reduces neointima formation in animal models $[10,13,14]$. However, none of the compounds could be clinically used in patients to prevent restenosis after coronary interventions due to pharmacological constraints or the lack of applicability.

Here we tested a small cyclic peptide integrin $\alpha_{\mathrm{v}}$ antagonist in vitro on its effects on human coronary SMCs. Our studies reveal a critical role for $\alpha_{\mathrm{v}}$-containing integrins in haptotaxis and chemotaxis migration of SMC on vitronectin. The blockage of SMC migration upon integrin $\alpha_{v}$ inhibition is accompanied by reduced integrin-initiated cellular signaling events involving the focal adhesion kinase. Interference with $\alpha_{\mathrm{v}}$-containing integrins not only blunts the motility, but also down-modulates the invasive capabilities of SMC by reducing MMP2 secretion. Together, our data suggest that $\alpha_{\mathrm{v}}$-directed cyclic peptide antagonists have the potential to abrogate smooth muscle cell migration in vivo and that such inhibitors could be clinically applied to humans to prevent restenosis.

\section{Results}

Integrin $\alpha_{v}$ expression in human coronary artery smooth muscle cells

Smooth muscle cells are known to express a variety of adhesion molecules, however, expression of particu- lar integrin heterodimers can vary depending on the origin of the cells [15]. To analyze the presence of integrins $\alpha_{v} \beta_{3}$ and $\alpha_{v} \beta_{5}$ in human coronary artery smooth muscle cells (hCASMCs), cells were analyzed by flow cytometry (Fig. 1A). Compared to cells incubated with an isotype-matched control antibody, both $\alpha_{\mathrm{v}} \beta_{3^{-}}$as well as $\alpha_{v} \beta_{5}$-directed monoclonal antibodies recognized the primary hCASMC demonstrating the presence of the respective integrins on the cell surface. To investigate the subcellular localization of the $\alpha_{v} \beta_{3}$ and the $\alpha_{\mathrm{v}} \beta_{5}$ integrin heterodimers in attached hCASMC, immunofluorescence staining of fixed samples was performed with monoclonal anti-integrin $\alpha_{\mathrm{v}} \beta_{3}$ or $\alpha_{\mathrm{v}} \beta_{5}$ antibodies, respectively, and phalloidin-TRITC. Confocal microscopy demonstrated that both integrins were localized predominantly to focal adhesions and focal contact points at the cell-substrate interface (Fig. 1B). In particular, the tips and leading lamellae of cell protrusions were found to contain clustered integrins. In addition, the integrin $\alpha_{\mathrm{v}}$-rich structures were organizing centers of the cytoplasmic actin, as bundles of polymerized actin emanated from there (Fig. 1B). Together, these data demonstrated that integrin $\alpha_{\mathrm{v}} \beta_{3}$ as well as $\alpha_{\mathrm{v}} \beta_{5}$ were expressed in hCASMC and had a functional distribution.

\section{Vitronectin stimulates focal adhesion kinase and migration of smooth muscle cells}

A physiologic ligand of smooth muscle cell integrins $\alpha_{v} \beta_{3}$ and $\alpha_{v} \beta_{5}$ is the extracellular matrix protein vitronectin. Clearly, immobilized vitronectin stimulated hCASMC migration in a dose-dependent manner in modified Boyden chamber haptotaxis assays (Fig. 2A). Biochemically, engagement of $\alpha_{v}$ integrins by plating cells onto a vitronectin matrix increased tyrosine phosphorylation of several proteins with an apparent molecular weight of $\sim 44, \sim 60$, and $\sim 120 \mathrm{kDa}$ (Fig. $2 \mathrm{~B}$ ). The most prominent increase in tyrosine phosphorylation was observed for a protein with $\sim 120 \mathrm{kDa}$ apparent molecular weight (Fig. 2B). Upon re-probing of this membrane with antibodies directed against focal adhesion kinase, a protein band of similar size was labeled (data not shown). To unambiguously identify the protein, immunoprecipitations with anti-FAK antibodies were carried out. After electrophoresis and blotting, the samples were probed with anti-phosphotyrosine antibodies (Fig. 2C). Indeed, only upon replating of the hCASMC onto vitronectin, but not in suspended cells or upon replating onto poly-L-lysine, a strong increase in tyrosine phosphorylation of FAK was observed (Fig. 2C; upper panel). In addition, phosphospecific antibodies directed against the FAK autophosphorylation site at tyrosine 397 showed an even more dramatic increase in tyrosine phosphorylation upon vitronectin replating, indicating that FAK kinase 

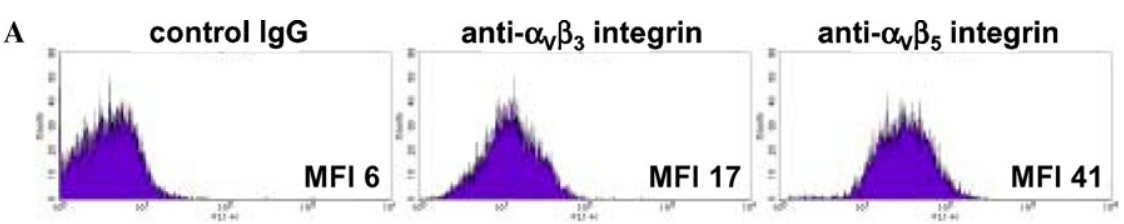

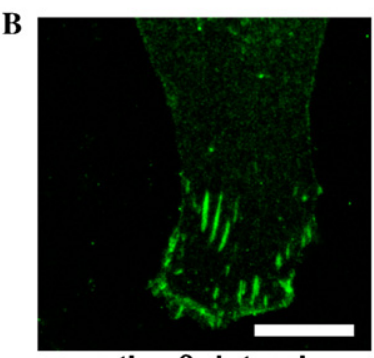

anti- $\alpha_{v} \beta_{3}$ integrin

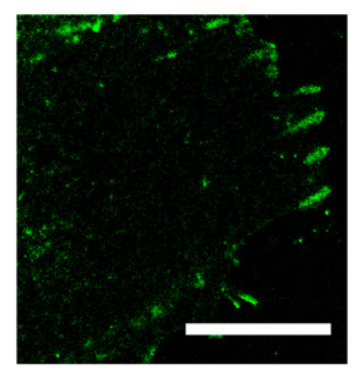

anti- $\alpha_{v} \beta_{5}$ integrin

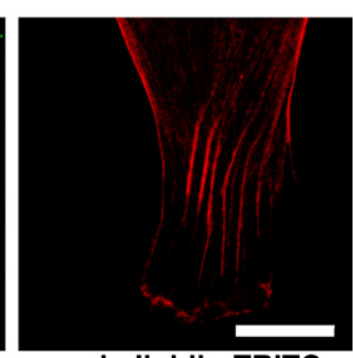

phalloidin-TRITC

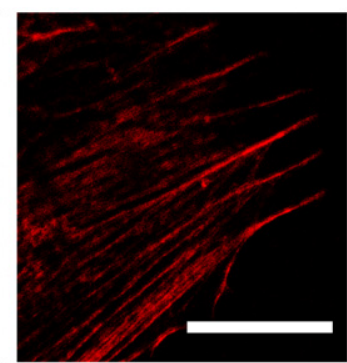

phalloidin-TRITC

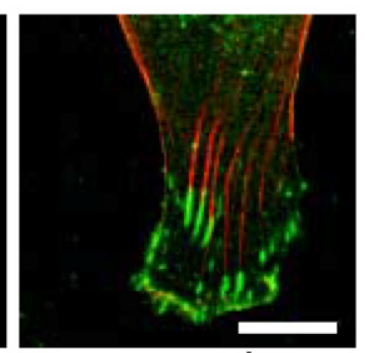

merged

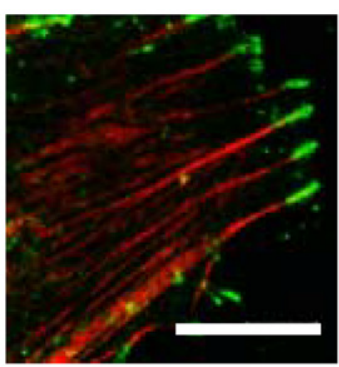

merged

Fig. 1. Integrin $\alpha_{v} \beta_{3}$ and $\alpha_{v} \beta_{5}$ expression and localization in primary human coronary artery smooth muscle cells. (A) Flow cytometric analysis of hCASMC stained with monoclonal antibodies directed against integrin $\alpha_{\mathrm{v}} \beta_{3}$, integrin $\alpha_{\mathrm{v}} \beta_{5}$, or an isotype-matched control antibody. Mean fluorescence intensity (MFI) of each sample is indicated. (B) Immunofluorescence co-staining of integrin $\alpha_{\mathrm{v}} \beta_{3}$ or integrin $\alpha_{\mathrm{v}} \beta_{5}$ and actin in hCASMC. Cells growing on gelatine-coated glass coverslips were fixed with ice-cold acetone, co-stained with the indicated FITC-labeled monoclonal antibodies and phalloidin-TRITC, and images were recorded with a confocal microscope. Bars represent $20 \mu \mathrm{m}$.

activity was increased (Fig. 2C; middle panel). Importantly, re-probing of the membrane with anti-FAK antibodies demonstrated comparable amounts of immunoprecipitated FAK in each sample (Fig. 2C; lower panel). These results demonstrated that vitronectin is a potent stimulus of hCASMC haptotaxis migration and FAK signaling.

To further verify that FAK signaling and enhanced tyrosine phosphorylation upon vitronectin binding occur in the context of integrin-rich focal adhesion sites, hCASMCs were plated for $45 \mathrm{~min}$ onto VN or PL, fixed, and stained for phosphotyrosine-containing proteins. Importantly, VN not only stimulated cell spreading and the generation of numerous peripheral focal adhesions as marked by the presence of vinculin (data not shown), but also induced strong tyrosine phosphorylation at these sites (Fig. 2D). In contrast, cells plated onto PL did not show accumulation of tyrosine phosphorylated proteins in focal attachment sites and tyrosine phosphorylation was only detected in a perinuclear region (Fig. 2D). These data support the view that binding to vitronectin triggers tyrosine phosphorylation events at focal adhesion sites that are connected to FAK stimulation.
Vitronectin-stimulated signaling events and cell migration are blocked by integrin $\alpha_{v}$ inhibition

To test the functional relevance of $\alpha_{\mathrm{v}}$ integrins in vitronectin-stimulated cellular events, we employed a small peptidomimetic inhibitor of integrin $\alpha_{\mathrm{v}}[16,17]$ and replated hCASMC onto vitronectin. Importantly, treatment with the $\alpha_{\mathrm{v}}$ inhibitor led to a dose-dependent decrease in overall tyrosine phosphorylation of FAK (Fig. 3A; upper panel). Already $1 \mu \mathrm{M}$ of the integrin $\alpha_{v}$ inhibitor caused a significant reduction in tyrosine phosphorylation, and a concentration of $10 \mu \mathrm{M}$ completely limited FAK tyrosine phosphorylation to background levels (Fig. 3A; upper panel). Re-probing of the membrane with monoclonal antiFAK antibodies demonstrated equal amounts of FAK protein in all samples (Fig. 3A; lower panel). Furthermore, modified Boyden chamber haptotaxis assays in the presence of the $\alpha_{\mathrm{v}}$ inhibitor demonstrated that vitronectin-stimulated migration of hCASMC was blocked in a dose-dependent manner by the compound (Fig. 3B). Clearly, the decrease in haptotaxis paralleled the reduced tyrosine phosphorylation of FAK, as inhibitor concentrations of $10 \mu \mathrm{M}$ resulted 
A

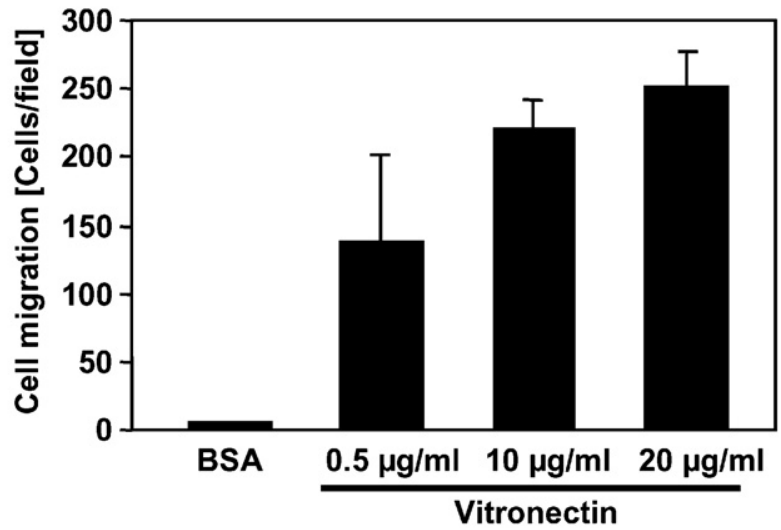

B

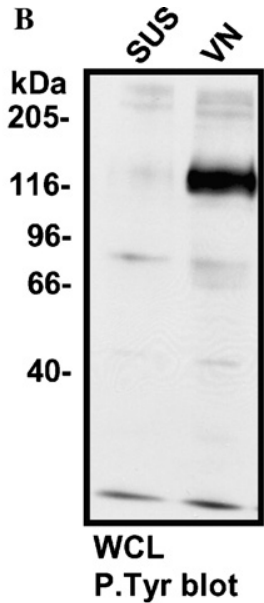

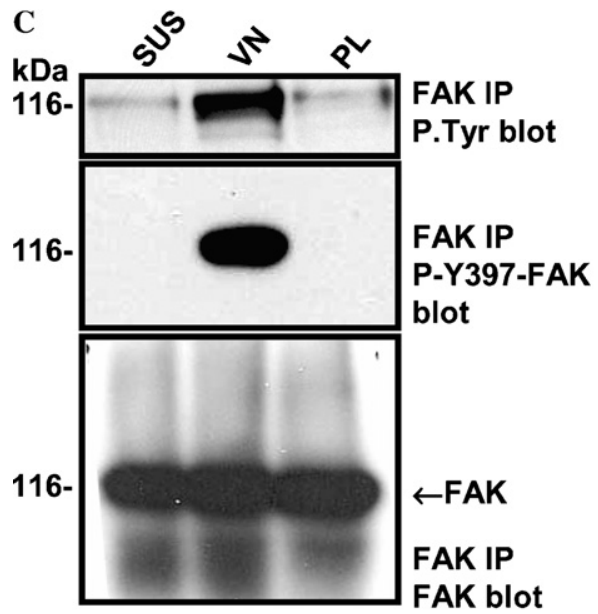
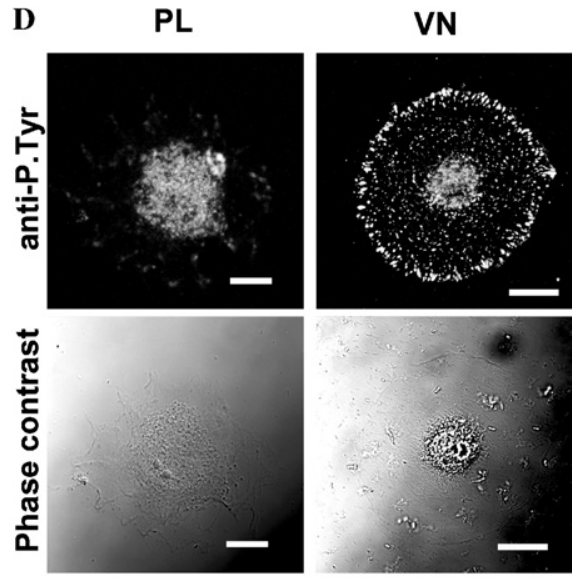

Fig. 2. Vitronectin stimulates haptotaxis and focal adhesion kinase phosphorylation in hCASMC. (A) Haptotaxis of hCASMC was analyzed in modified Boyden chambers coated with the indicated amounts of vitronectin. After $3 \mathrm{~h}$, transmigrated cells were fixed, stained, and counted in 5 random fields per chamber. Bars represent mean cell numbers/field \pm standard deviation from three to five chambers. (B) Serum-starved hCASMCs were either kept in suspension (SUS) or replated onto vitronectin-coated $(10 \mu \mathrm{g} / \mathrm{ml})$ plates (VN) for $45 \mathrm{~min}$. Whole cell lysates (WCL) were analyzed further by Western blotting with anti-phosphotyrosine (P.Tyr) antibodies. (C) Serum-starved SMCs were kept in suspension (SUS) or replated onto either vitronectin- (VN; $10 \mu \mathrm{g} / \mathrm{ml}$ ) or poly-L-lysine (PL; $20 \mu \mathrm{g} / \mathrm{ml}$ ) -coated plates for $45 \mathrm{~min}$. FAK was immunoprecipitated (IP) and IPs were analyzed with P.Tyr antibodies (upper panel), probed with phospho-specific antibodies to the auto-phosphorylation site of FAK at Tyr-397 (middle panel), or a monoclonal anti-FAK antibody (lower panel). (D) Serum-starved hCASMCs were replated as in (C), fixed after 45 min, and processed for immunofluorescence microscopy with monoclonal antibodies against phospho-tyrosine and Cy3-coupled secondary antibodies. Samples were viewed with a confocal laser scanning microscope. Bars represent $20 \mu \mathrm{m}$.

in a decrease in haptotaxis of more than $80 \%$. Besides immobilized extracellular matrix proteins, migration of hCASMC can also be stimulated by soluble growth factors such as PDGF-BB. To test whether $\alpha_{\mathrm{v}}$ integrins also contribute to PDGF-BB-induced chemotaxis migration, hCASMCs were employed in a chemotaxis migration assay in the absence or presence of the integrin $\alpha_{\mathrm{v}}$ inhibitor (Fig. 3C). Maximal chemotaxis of primary hCASMC was observed between 50 and $100 \mathrm{ng} / \mathrm{ml}$ PDGF-BB (Laser and Varadarajulu, unpublished observations). However, when $\alpha_{\mathrm{v}}$ integrins were blocked, chemotaxis stimulated by $50 \mathrm{ng} / \mathrm{ml}$ PDGFBB was almost completely abolished, when cells were observed in the presence of $10-100 \mu \mathrm{g} / \mathrm{ml}$ of the inhibitor (Fig. 3C).
Integrin $\alpha_{v}$ inhibition blocks tyrosine phosphorylation events at focal contacts

FAK activity and tyrosine phosphorylation events at cell-matrix contact points are required for the dynamic regulation of focal adhesion turnover [18]. To monitor the influence of integrin $\alpha_{\mathrm{v}}$ inhibition on tyrosine phosphorylation events at focal adhesions, SMCs were plated for $45 \mathrm{~min}$ onto vitronectin in the absence or presence of the integrin $\alpha_{\mathrm{v}}$ inhibitor. As observed before, cells adhering to $\mathrm{VN}$ showed focal accumulation of phosphotyrosine-containing proteins predominantly at the cell periphery (Fig. 4). In contrast, cells plated onto $\mathrm{VN}$ in the presence of $10 \mu \mathrm{M}$ integrin $\alpha_{\mathrm{v}}$ inhibitor almost completely lacked discrete focal 

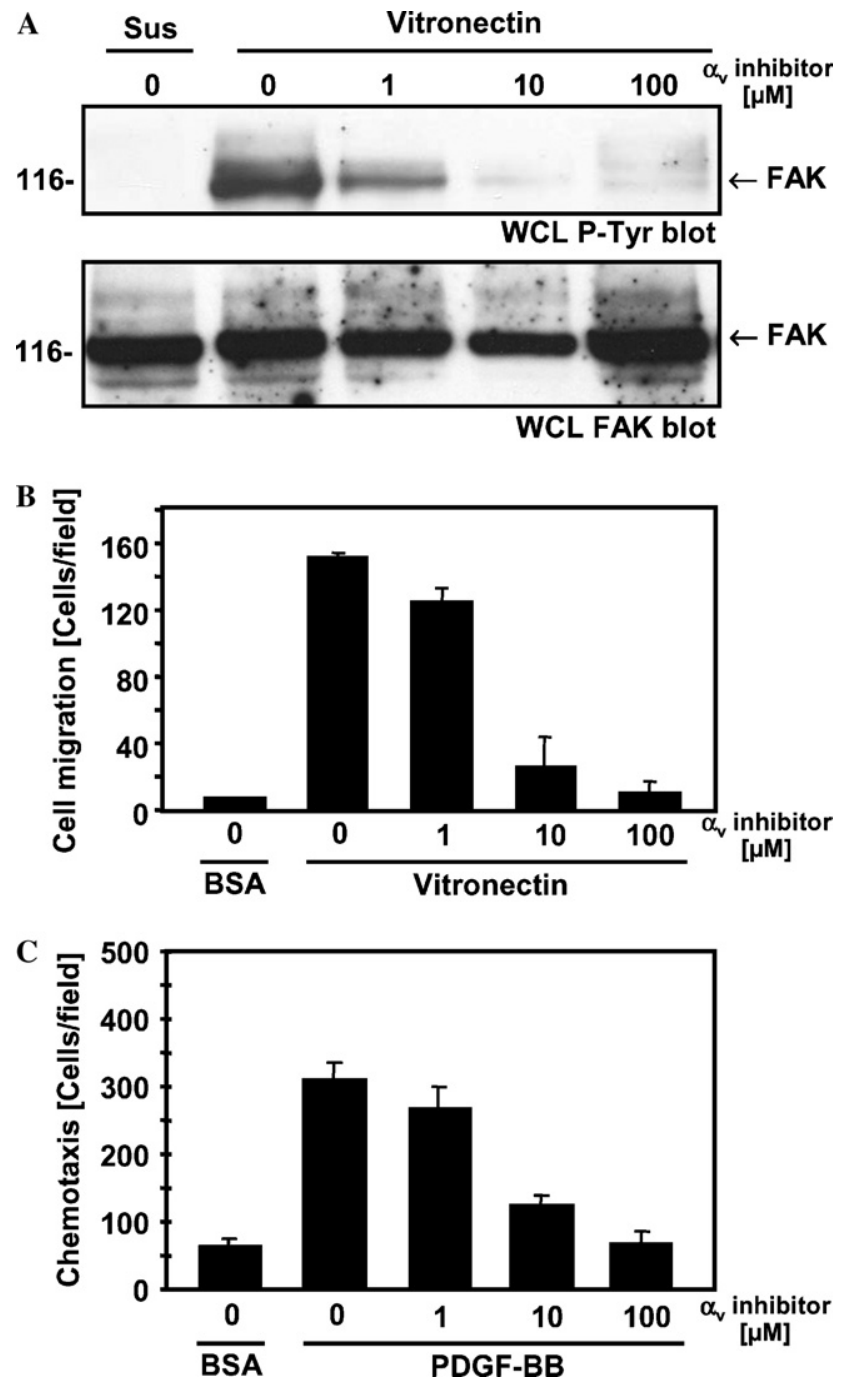

Fig. 3. Vitronectin-stimulated signaling events and cell migration are blocked by integrin $\alpha_{\mathrm{v}}$ inhibition. (A) Serum-starved hCASMCs were either kept in suspension (SUS) or replated onto vitronectincoated $(5 \mu \mathrm{g} / \mathrm{ml})$ plates for $45 \mathrm{~min}$ in the presence of the indicated concentrations of the integrin $\alpha_{\mathrm{v}}$ inhibitor. Cells were lysed and whole cell lysates (WCL) were analyzed by Western blotting with anti-phosphotyrosine (P.Tyr) antibodies (upper panel). Membranes were re-probed with monoclonal anti-FAK antibodies (lower panel). (B) Haptotaxis of hCASMC was analyzed in modified Boyden chambers coated with BSA or $5 \mu \mathrm{g} / \mathrm{ml}$ vitronectin in the presence of the indicated concentrations of the integrin $\alpha_{\mathrm{v}}$ inhibitor. Bars represent mean cell numbers per field of view \pm standard deviation from three chambers. (C) hCASMC chemotaxis was induced by $50 \mathrm{ng} / \mathrm{ml}$ PDGF-BB in the presence of the indicated concentrations of the integrin $\alpha_{\mathrm{v}}$ inhibitor. Bars represent mean cell numbers per field of view \pm standard deviation from three chambers.

tyrosine phosphorylation (Fig. 4). The inhibitor also severely interfered with the spreading of the cells on the extracellular matrix substrate, a process known to require remodeling of focal adhesions and a prerequisite for cell migration.

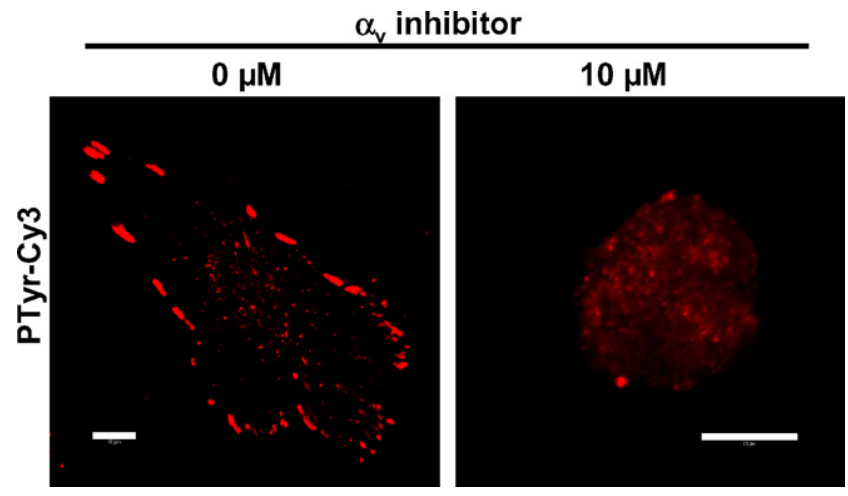

Fig. 4. Integrin $\alpha_{v}$ inhibition blocks vitronectin-triggered tyrosine phosphorylation events at focal contacts. Serum-starved SMCs were replated onto vitronectin-coated $(5 \mu \mathrm{g} / \mathrm{ml})$ glass coverslips in the absence or presence of $10 \mu \mathrm{M}$ integrin $\alpha_{v}$ inhibitor. Attached cells were fixed after $45 \mathrm{~min}$, and processed for immunofluorescence microscopy with monoclonal antibodies against phospho-tyrosine and Cy3coupled secondary antibodies. Samples were viewed with a confocal laser scanning microscope. Bars represent $10 \mu \mathrm{m}$.

\section{Targeting integrin $\alpha_{v}$ impairs invasive motility of $h C A S M C$}

Migration of smooth muscle cells from the media of injured vessels to the intima requires invasive motility in a three-dimensional environment. To more closely mimic this situation, hCASMC invasive motility through a reconstituted basement membrane (Matrigel) was investigated. Therefore, modified Boyden chambers were covered with a layer of growth-factor-reduced Matrigel at different concentrations and the ability of the cells to migrate through this three-dimensional matrix was measured (Fig. 5A). Primary hCASMCs were able to invade through layers of up to $10 \mu \mathrm{g}$ Matrigel per chamber. At higher Matrigel concentrations, invasion of the cells was severely impaired (Fig. 5A). When invasion assays through layers of $10 \mu \mathrm{g}$ Matrigel per chamber were conducted in the presence of the integrin $\alpha_{\mathrm{v}}$ inhibitor, invasive motility of hCASMC was strongly reduced (Fig. 5B). This reduction in Matrigel invasion correlated with a decreased secretion of matrix metalloproteases from hCASMC cultured in the presence of the integrin $\alpha_{\mathrm{v}}$ inhibitor (Fig. 5C). In particular, MMP-2 secretion was reduced in a dose-dependent manner with increasing concentrations of the inhibitor leading to lower levels of secreted protease activity as detected by gelatine zymography (Fig. 5C). Together, these results suggest that blocking of $\alpha_{\mathrm{v}}$ integrins and integrin-dependent signals affects motility as well as the invasive capabilities of hCASMC.

\section{Materials and methods}

Cells and cell culture. Primary human coronary artery smooth muscle cells (hCASMCs) were obtained from Clonetics (San Diego, 
A

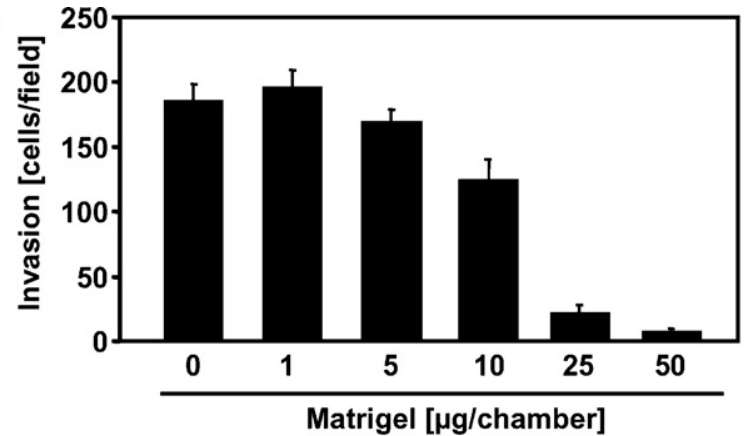

B

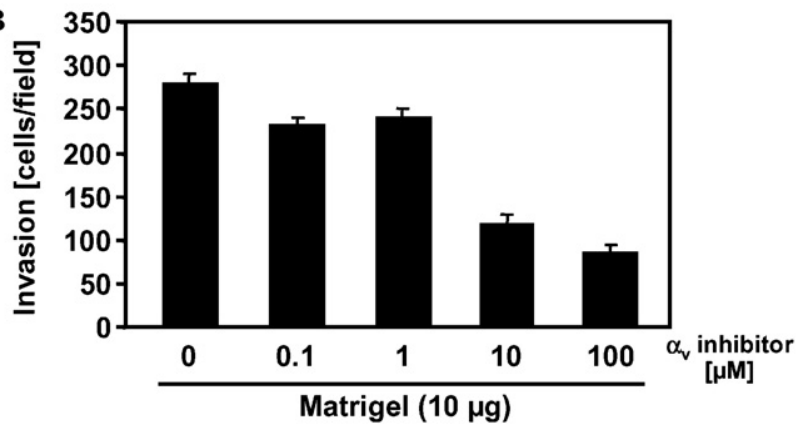

C SMC culture supernatant

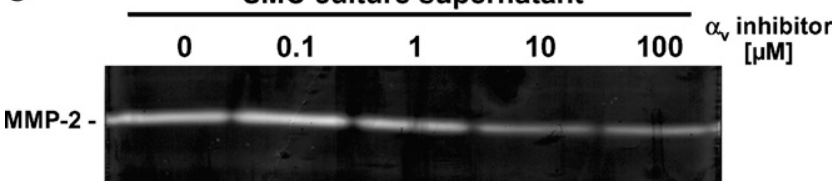

Fig. 5. Matrigel invasion and MMP secretion by hCASMC are blocked by the integrin $\alpha_{v}$ inhibitor. (A) Membranes of modified Boyden chambers were overlaid with the indicated amounts of a reconstituted basement membrane (Matrigel). Invasion of hCASMC towards the fetal bovine serum $(5 \%)$ containing lower compartment was allowed for $24 \mathrm{~h}$. Then cells that had invaded the lower compartment were fixed, stained, and counted. Bars represent mean values \pm standard deviation from five random fields taken from each of three separate chambers. (B) Invasion of hCASMC through Matrigel (10 $\mu \mathrm{g}$ per chamber) was analyzed as above in the presence or absence of the indicated concentrations of integrin $\alpha_{v}$ inhibitor. Bars represent mean values \pm standard deviation from five random fields taken from each of three separate chambers. (C) hCASMCs were plated onto vitronectin-coated dishes for $16 \mathrm{~h}$ in the presence or absence of the indicated concentrations of integrin $\alpha_{v}$ inhibitor. Conditioned medium from each culture was analyzed by gelatine zymography.

CA) and cultured on gelatine-coated culture dishes between passage 2 and 8 in smooth muscle basal medium (Cambrex Biosciences, Walkersville, MD) supplemented with SmGM2 (Clonetics) at $37^{\circ} \mathrm{C}$ with $5 \%$ $\mathrm{CO}_{2}$. More than $95 \%$ of the cells in all passages stained positive for the smooth muscle-specific isoform of actin by immunofluorescence staining (data not shown). In some cases, cells were serum starved using DMEM with $0.5 \%$ fetal bovine serum (FBS). Where indicated, serum-starved cells were detached by limited trypsin treatment $(0.025 \%$ trypsin/0.5 mM EDTA for $2 \mathrm{~min}$ ), collected in the presence of soybean trypsin inhibitor $(0.25 \mathrm{mg} / \mathrm{ml}$ in DMEM $)$, resuspended in DMEM with $0.25 \%$ BSA (suspension medium), and held in suspension for $45 \mathrm{~min}$ at $37^{\circ} \mathrm{C}$ prior to use.

Antibodies and reagents. Fluorescein-conjugated monoclonal antibodies (mAb) against integrin $\alpha_{\mathrm{v}} \beta_{3}$ (clone LM609), integrin $\alpha_{\mathrm{v}} \beta_{5}$ (clone
P1F6), and control $\mathrm{IgG}_{1}$ (clone DD7) were from Chemicon (Temecula, $\mathrm{CA}$ ), mAb against smooth muscle cell actin (clone 1A4) was from Sigma (Taufkirchen, Germany), mAb against phosphotyrosine (P.Tyr) (clone 4G10) was from Upstate Biotechnology (Lake Placid, NY), and $\mathrm{mAb}$ (clone 77) and polyclonal antibodies (A17) against FAK were from BD Biosciences (Heidelberg, Germany) or Santa Cruz Biotechnology (Santa Cruz, CA), respectively; phospho-specific polyclonal antibodies against P-Y397 FAK were from QCB BioSource International (Hopkinton, MA). Peroxidase-conjugated protein A was from ICN Biochemicals (Eschwege, Germany), peroxidase-conjugated rabbit anti-mouse antibodies were from DaKo (Glastrup, DK). Alexa Fluor-546 phalloidin was from Molecular Probes (Eugene, OR), while Cy2- and Cy3-conjugated goat-anti-mouse antibodies were from Jackson Immunoresearch (West Grove, PA). The integrin $\alpha_{\mathrm{v}}$ inhibitor (EMD121974) was a generous gift from Merck KG, Darmstadt, Germany, and characterized previously $[16,17]$.

Flow cytometry. Suspended hCASMCs were taken up in PBS, $0.25 \% \mathrm{BSA}$ at $8 \times 10^{5}$ cells $/ \mathrm{ml}$ and $200 \mu \mathrm{l}$ of cell suspension was incubated with $2 \mu \mathrm{g}$ FITC-labeled mAb against integrin $\alpha_{\mathrm{v}} \beta_{3}$, integrin $\alpha_{\mathrm{v}} \beta_{5}$, or an isotype-matched control antibody on ice for $45 \mathrm{~min}$. Cells were washed three times with PBS, $0.25 \%$ BSA and analyzed using a FACSCalibur (Becton-Dickinson).

Immunofluorescence staining. Suspended hCASMCs $\left(2 \times 10^{4}\right)$ were plated onto acid-washed glass coverslips coated overnight at $4{ }^{\circ} \mathrm{C}$ with $5 \mu \mathrm{g} / \mathrm{ml}$ vitronectin and allowed to attach for $45 \mathrm{~min}$. Cells were fixed with ice-cold acetone for $15 \mathrm{~min}$, washed with PBS, and incubated in PBS, $10 \%$ calf serum, and $0.2 \%$ saponin (blocking solution) for $5 \mathrm{~min}$. Primary antibodies in blocking solution were added for $45 \mathrm{~min}$, samples were washed three times with blocking solution, and incubated with fluorescent-dye-coupled secondary reagents for $45 \mathrm{~min}$ at room temperature in the dark. After three washes with PBS, samples were embedded in mounting medium (DaKo, Glastrup, DK) and analyzed with a LSM510 confocal laser scanning microscope (Zeiss, Oberkochen, Germany).

Cell migration and invasion assays. Cell migration and Matrigel invasion assays were performed as described [19]. Briefly, Millicell chambers ( $12 \mathrm{~mm}$ diameter; $8 \mu \mathrm{m}$ pores; Millipore, Bedford, MA) were coated on the lower side of the membrane with the indicated concentrations of human vitronectin (Chemicon) for haptotaxis assays or coated with $5 \mu \mathrm{g} / \mathrm{ml}$ vitronectin on both sides for chemotaxis assays for $2 \mathrm{~h}$ at room temperature. In control chambers, the membrane was coated with $0.25 \%$ (BSA) in DMEM. For invasion assays, the indicated amounts of growth-factor-reduced Matrigel (BD Biosciences, Bedford, MA) were polymerized as described on top of the membrane [19]. $1.5 \times 10^{5}$ suspended hCASMCs in migration media (DMEM with $0.5 \% \mathrm{BSA}$ ) were added to the upper compartment and the chambers were placed into 24-well culture dishes containing $0.4 \mathrm{ml}$ migration media for integrin-stimulated haptotaxis, DMEM with the indicated concentrations of platelet-derived growth factor BB (PDGF-BB; Upstate Biotechnology, Lake Placid, NY) for chemotaxis, or 5\% FBS for invasion assays, respectively. After $3 \mathrm{~h}$ (haptotaxis and chemotaxis) or $24 \mathrm{~h}$ (Matrigel invasion) at $37^{\circ} \mathrm{C}$, cells on the upper membrane surface were removed by a cotton tip applicator, chambers were washed with PBS, and migratory cells on the lower membrane surface were fixed by treatment with $40 \%$ methanol $/ 25 \%$ acetic acid. Cells were stained $(0.1 \%$ crystal violet, $0.1 \mathrm{M}$ borate, $\mathrm{pH} 9.0$, and $2 \%$ ethanol) and migration values were determined by counting cells in 5 fields of view $(20 \times)$ per chamber. Mean values were obtained from at least three individual chambers for each experimental point per assay.

Replating assay. Ten centimetre cell culture dishes were coated at $4{ }^{\circ} \mathrm{C}$ for $16 \mathrm{~h}$ with the indicated concentrations of human vitronectin or $10 \mu \mathrm{g} / \mathrm{ml}$ poly-L-lysine (Sigma). Suspended hCASMCs were allowed to attach to the substrates for $45 \mathrm{~min}$. In some experiments, the $\alpha_{\mathrm{v}}$ integrin inhibitor was added at the indicated concentrations to the suspended cells and was present during the attachment phase. After 45 min attachment, cells were washed once with ice-cold PBS and lysed. 
Cell lysis immunoprecipitation, Western blotting, and gelatine zymography. Cells were solubilized in a modified RIPA lysis buffer containing $1 \%$ Triton X-100, $1 \%$ sodium deoxycholate, and $0.1 \%$ SDS [20]. Immunoprecipitations, SDS-PAGE, Western blotting, and gelatine zymography were performed as described $[19,21]$.

\section{Discussion}

Smooth muscle cell migration is a key process in the pathophysiology of restenosis. In the present study, we were able to show the ability of a small cyclic peptide integrin $\alpha_{\mathrm{v}}$ inhibitor, suitable for drug-eluting stents, to prevent human coronary artery smooth muscle cell migration and invasion. Inhibition of $\alpha_{v}$-containing integrins strongly reduced haptotaxis and chemotaxis motility. The cellular responses that were blocked by the integrin $\alpha_{v}$ inhibitor involved vitronectin-initiated stimulation of the focal adhesion kinase (FAK) as well as the release of MMP-2, two enzymes involved in cell motility and tissue invasion [18].

Although several studies suggest an involvement of $\alpha_{\mathrm{v}}$-containing integrins, and in particular integrin $\alpha_{\mathrm{v}} \beta_{3}$, in restenosis and smooth muscle cell migration $[5,10,11,13,14,22]$ in vivo and in vitro, it is still unclear how blockage of this particular ECM receptor translates into an inhibition of SMC migration induced by various stimuli. Our results point to a critical role of integrintriggered cellular signaling events involving FAK in the regulation of hCASMC migration. This protein tyrosine kinase (PTK) has been implicated in the control of cell migration in a number of adherent cell types including fibroblasts [23], endothelial cells [24], and epithelial cells [25]. In response to integrin engagement by extracellular matrix proteins, FAK is rapidly activated and undergoes autophosphorylation at a critical tyrosine residue, Tyr-397, initiating an SH2 domain-dependent association with c-Src and the concomitant recruitment of additional proteins into a FAK-Src complex [26]. The activity of this complex seems to be of critical importance for the dynamic processes of focal adhesion assembly and disassembly that are essential for cell motility. As blockage of $\alpha_{\mathrm{v}}$ integrins dramatically diminishes tyrosine phosphorylation at focal adhesion sites and in particular the phosphorylation of FAK, this observation helps to explain the profound negative effect of the integrin $\alpha_{v}$ inhibitor on vitronectin-stimulated cell motility.

Apart from the haptotactic stimulation through matrix proteins, a variety of growth factors have been described to participate in the process of SMC stimulation during restenosis contributing to the phenotypic change into proliferating, migrating, and synthesizing cells. One of the most important factors seems to be PDGF-BB. Interestingly, while the inhibition of vitronectin-stimulated haptotaxis by an $\alpha_{\mathrm{v}}$ integrin inhibitor is not unexpected, the inhibition of PDGFtriggered chemotaxis by the same compound is somewhat more intriguing. The data suggest that $\alpha_{\mathrm{v}}$ integrins play a key regulatory role in both haptotactic and chemotactic migration of hCASMC. It has been demonstrated previously that growth factor stimulation efficiently elicits cellular responses only in the presence of integrin ligation (for review [27]). Accordingly, disruption of integrin-binding leads to inefficient growth factor stimulation up to a complete suppression of growth factor-triggered signals as seen, for example, in suspended cells [28]. In addition, PDGF receptors can associate with the $\alpha_{v} \beta_{3}$ integrin leading to an enhanced signaling through PDGF [29]. In this context, it is interesting to note that FAK has been reported to serve as a molecular connection between growth factor- and integrin-initiated signals. Indeed, FAK not only is recruited to integrin-rich focal adhesion sites upon cell contact with extracellular matrix proteins [30], but is also found in a complex with activated growth factor receptors such as the epidermal growth factor receptor or the PDGF receptor [31]. A PDGF receptor-FAK complex can be detected upon PDGF-BB stimulation of rat smooth muscle cells and interference with FAK activation by overexpression of the endogenous FAK inhibitor FRNK blocks FAK-PDGF receptor association and SMC migration [32]. Therefore, $\alpha_{\mathrm{v}}$ integrin inhibitors that interfere with FAK activation are able to attenuate both haptotaxis and chemotaxis of hCASMC, making $\alpha_{\mathrm{v}}$ integrins attractive targets to block neointima formation in vivo.

Cell migration in vivo takes place in a three-dimensional environment and requires cells to overcome extracellular matrix barriers. In vitro Matrigel invasion is a convenient way to measure the invasive capabilities of isolated cells. Interestingly, hCASMCs are able to overcome a Matrigel barrier in modified Boyden migration chambers. However, inhibition of $\alpha_{\mathrm{v}}$ integrins reduced in vitro Matrigel invasion of hCASMC to about 30\% of that seen in untreated cells. The impaired invasion of the inhibitor treated cells was accompanied by a reduced secretion of MMP-2 from hCASMC. Interestingly, an involvement of MMP-2 in invasive motility of mouse SMC has been described previously [33]. In vivo, MMP-2 is also strongly upregulated during intimal remodeling upon carotid artery ligation [34]. Importantly, in this model of experimental neointima induction, MMP-2 knock-out mice show strongly reduced intimal hyperplasia when compared to wildtype mice suggesting that MMP-2 is critically involved in vascular remodeling and restenosis [34]. Our results now provide a link between integrin $\alpha_{v}$ engagement and MMP-2 secretion, and imply that hCASMC stimulation by vitronectin not only initiates cell motility, but also modulates the invasive capabilities of these cells. It is 
interesting to note that FAK-dependent signaling is important for the invasive capabilities of transformed mouse fibroblasts and human carcinoma cells by positively contributing to MMP expression [19,21,35]. Therefore, interference with integrin $\alpha_{\mathrm{v}}$ engagement as well as FAK activation is likely to impair MMP-2 expression by hCASMC thereby attenuating the invasiveness of these cells. Taken together, our data support the view that $\alpha_{v}$-containing integrins play a key role in hCASMC motility and invasion by regulating FAK-dependent cellular signaling events connected to haptotaxis and chemotaxis migration as well as MMP-2 expression and Matrigel invasion. Therefore, application of $\alpha_{\mathrm{v}}$ inhibitors could be a promising approach to prevent restenosis and local intimal hyperplasia.

\section{Acknowledgments}

The authors thank A. Jonczyk (Merck, Darmstadt, Germany) for reagents, and G. Ertl and J. Hacker for helpful discussions. This study was supported by funds from the Interdisziplinäres Zentrum für klinische Forschung (Z-4/47) to M.L. and C.R.H, and the Deutsche Forschungsgemeinschaft (La 1063/3-1) to M.L.

\section{References}

[1] J. Rutanen, J. Markkanen, S. Yla-Herttuala, Gene therapy for restenosis: current status, Drugs 62 (2002) 1575-1585.

[2] T.C. Woods, A.R. Marks, Drug-eluting stents, Annu. Rev. Med. 55 (2004) 169-178.

[3] R. Virmani, A. Farb, Pathology of in-stent restenosis, Curr. Opin. Lipidol. 10 (1999) 499-506.

[4] M.H. Corjay, S.M. Diamond, K.L. Schlingmann, S.K. Gibbs, J.K. Stoltenborg, A.L. Racanelli, alphavbeta3, alphavbeta5, and osteopontin are coordinately upregulated at early time points in a rabbit model of neointima formation, J. Cell. Biochem. 75 (1999) 492-504.

[5] D. Panda, G.C. Kundu, B.I. Lee, A. Peri, D. Fohl, I. Chackalaparampil, B.B. Mukherjee, X.D. Li, D.C. Mukherjee, S. Seides, J. Rosenberg, K. Stark, A.B. Mukherjee, Potential roles of osteopontin and alphaVbeta3 integrin in the development of coronary artery restenosis after angioplasty, Proc. Natl. Acad. Sci. USA 94 (1997) 9308-9313.

[6] M. Hoshiga, C.E. Alpers, L.L. Smith, C.M. Giachelli, S.M. Schwartz, Alpha-v beta-3 integrin expression in normal and atherosclerotic artery, Circ. Res. 77 (1995) 1129-1135.

[7] S.A. Mousa, Vitronectin receptors in vascular disorders, Curr. Opin. Invest. Drugs 3 (2002) 1191-1195.

[8] N. Kipshidze, M.B. Leon, M. Tsapenko, R. Falotico, G.A. Kopia, J. Moses, Update on sirolimus drug-eluting stents, Curr. Pharm. Des. 10 (2004) 337-348.

[9] R. Virmani, G. Guagliumi, A. Farb, G. Musumeci, N. Grieco, T. Motta, L. Mihalcsik, M. Tespili, O. Valsecchi, F.D. Kolodgie, Localized hypersensitivity and late coronary thrombosis secondary to a sirolimus-eluting stent: should we be cautious? Circulation 109 (2004) 701-705.

[10] S.S. Srivatsa, L.A. Fitzpatrick, P.W. Tsao, T.M. Reilly, D.R. Holmes Jr., R.S. Schwartz, S.A. Mousa, Selective alpha v beta 3 integrin blockade potently limits neointimal hyperplasia and lumen stenosis following deep coronary arterial stent injury: evidence for the functional importance of integrin alpha $v$ beta 3 and osteopontin expression during neointima formation, Cardiovasc. Res. 36 (1997) 408-428.

[11] J.H. Baron, E.P. Moiseeva, D.P. de Bono, K.R. Abrams, A.H. Gershlick, Inhibition of vascular smooth muscle cell adhesion and migration by c7E3 Fab (abciximab): a possible mechanism for influencing restenosis, Cardiovasc. Res. 48 (2000) 464-472.

[12] T.V. Byzova, R. Rabbani, S.E. D’Souza, E.F. Plow, Role of integrin alpha(v)beta3 in vascular biology, Thromb. Haemost. 80 (1998) 726-734.

[13] H. Matsuno, J.M. Stassen, J. Vermylen, H. Deckmyn, Inhibition of integrin function by a cyclic RGD-containing peptide prevents neointima formation, Circulation 90 (1994) 2203-2206.

[14] G.G. Bishop, J.A. McPherson, J.M. Sanders, S.E. Hesselbacher, M.J. Feldman, C.A. McNamara, L.W. Gimple, E.R. Powers, S.A. Mousa, I.J. Sarembock, Selective alpha(v)beta(3)-receptor blockade reduces macrophage infiltration and restenosis after balloon angioplasty in the atherosclerotic rabbit, Circulation 103 (2001) 1906-1911.

[15] E.P. Moiseeva, Adhesion receptors of vascular smooth muscle cells and their functions, Cardiovasc. Res. 52 (2001) 372-386.

[16] S.J. DeNardo, P.A. Burke, B.R. Leigh, R.T. O'Donnell, L.A. Miers, L.A. Kroger, S.L. Goodman, S. Matzku, A. Jonczyk, K.R. Lamborn, G.L. DeNardo, Neovascular targeting with cyclic RGD peptide (cRGDf-ACHA) to enhance delivery of radioimmunotherapy, Cancer Biother. Radiopharm. 15 (2000) 71-79.

[17] H.N. Lode, T. Moehler, R. Xiang, A. Jonczyk, S.D. Gillies, D.A. Cheresh, R.A. Reisfeld, Synergy between an antiangiogenic integrin alphav antagonist and an antibody-cytokine fusion protein eradicates spontaneous tumor metastases, Proc. Natl. Acad. Sci. USA 96 (1999) 1591-1596.

[18] C.R. Hauck, D.A. Hsia, D.D. Schlaepfer, The focal adhesion kinase - a regulator of cell migration and invasion, IUBMB Life 53 (2002) 115-119.

[19] C.R. Hauck, D.A. Hsia, X.S. Puente, D.A. Cheresh, D.D. Schlaepfer, FRNK blocks v-Src-stimulated invasion and experimental metastases without effects on cell motility or growth, EMBO J. 21 (2002) 6289-6302.

[20] F. Agerer, A. Michel, K. Ohlsen, C.R. Hauck, Integrin-mediated invasion of Staphylococcus aureus into human cells requires Src family protein tyrosine kinases, J. Biol. Chem. 278 (2003) 42524 42531.

[21] C.R. Hauck, T. Hunter, D.D. Schlaepfer, The v-Src SH3 domain facilitates a cell adhesion-independent association with FAK, J. Biol. Chem. 276 (2001) 17653-17662.

[22] M. Sajid, G.A. Stouffer, The role of alpha(v)beta3 integrins in vascular healing, Thromb. Haemost. 87 (2002) 187-193.

[23] D.J. Sieg, C.R. Hauck, D.D. Schlaepfer, Required role of focal adhesion kinase (FAK) for integrin-stimulated cell migration, J. Cell Sci. 112 (1999) 2677-2691.

[24] A.P. Gilmore, L.H. Romer, Inhibition of focal adhesion kinase (FAK) signaling in focal adhesions decreases cell motility and proliferation, Mol. Biol. Cell 7 (1996) 1209-1224.

[25] G.W. McLean, E. Avizienyte, M.C. Frame, Focal adhesion kinase as a potential target in oncology, Expert Opin. Pharmacother. 4 (2003) 227-234.

[26] D.D. Schlaepfer, M.A. Broome, T. Hunter, Fibronectin-stimulated signaling from a focal adhesion kinase-c-src complex: involvement of the Grb2, p130Cas, and Nck adaptor proteins, Mol. Cell. Biol. 17 (1997) 1702-1713.

[27] C.H. Damsky, D. Ilic, Integrin signaling: it's where the action is, Curr. Opin. Cell Biol. 14 (2002) 594-602.

[28] S. Miyamoto, H. Teramoto, J. Gutkind, K. Yamada, Integrins can collaborate with growth factors for phosphorylation of receptor tyrosine kinases and MAP kinase activation: roles of 
integrin aggregation and occupancy of receptors, J. Cell Biol. 135 (1996) 1633-1642.

[29] M. Schneller, K. Vuori, E. Ruoslahti, alphav beta 3 integrin associates with activated insulin and PDGFbeta receptors and potentiates the biological activity of PDGF, EMBO J. 16 (1997) 5600-5607.

[30] C.K. Klingbeil, C.R. Hauck, K.C. Jones, S.R. Reider, D.D. Schlaepfer, Targeting Pyk2 to $\beta 1$-integrin-containing focal contacts rescues fibronectin-stimulated signaling and haptotactic motility defects of focal adhesion kinase-null cells, J. Cell Biol. 152 (2001) 97-110.

[31] D.J. Sieg, C.R. Hauck, D. Ilic, C.K. Klingbeil, E. Schaefer, C.H. Damsky, D.D. Schlaepfer, FAK integrates growth factor and integrin signals to promote cell migration, Nat. Cell Biol. 2 (2000) 249-256.

[32] C.R. Hauck, D.A. Hsia, D.D. Schlaepfer, Focal adhesion kinase facilitates PDGF-BB-stimulated ERK2 activation required for chemotaxis migration of vascular smooth muscle cells, J. Biol. Chem. 275 (2000) 41092-41099.

[33] S. Kanda, M. Kuzuya, M.A. Ramos, T. Koike, K. Yoshino, S. Ikeda, A. Iguchi, Matrix metalloproteinase and alphavbeta3 integrin-dependent vascular smooth muscle cell invasion through a type I collagen lattice, Arterioscler. Thromb. Vasc. Biol. 20 (2000) 998-1005.

[34] M. Kuzuya, S. Kanda, T. Sasaki, N. Tamaya-Mori, X.W. Cheng, T. Itoh, S. Itohara, A. Iguchi, Deficiency of gelatinase A suppresses smooth muscle cell invasion and development of experimental intimal hyperplasia, Circulation 108 (2003) 13751381.

[35] D.A. Hsia, S.K. Mitra, C.R. Hauck, D.N. Streblow, J.A. Nelson, D. Ilic, S. Huang, E. Li, G.R. Nemerow, J. Leng, K.S. Spencer, D.A. Cheresh, D.D. Schlaepfer, Differential regulation of cell motility and invasion by FAK, J. Cell Biol. 160 (2003) 753-767. 